\title{
Bimodal Locomotion Elicited by Electrical Stimulation of the Midbrain in the Salamander Notophthalmus viridescens
}

\author{
Jean-Marie Cabelguen, ${ }^{1}$ Céline Bourcier-Lucas, ${ }^{2}$ and Réjean Dubuc ${ }^{2,3}$ \\ ${ }^{1}$ Institut National de la Santé et de la Recherche Medicale, Equipe 0358, Physiopathologie des Réseaux Neuronaux Médullaires, Institut François Magendie, \\ 33077 Bordeaux Cedex, France, ${ }^{2}$ Centre de Recherche en Sciences Neurologiques, Université de Montréal, Montréal, Québec, Canada H3C 3J7, and \\ ${ }^{3}$ Département de Kinanthropologie, Université du Québec à Montréal, Montréal, Québec, Canada H3C 3P8
}

\begin{abstract}
The present experiments were designed to identify the mesencephalic locomotor region (MLR) in the salamander. An in vitro semi-intact preparation from a decerebrate adult salamander (Notophthalmus viridescens) was developed in which the locomotor activities were monitored from electromyographic and video recordings. The results show that the two locomotor modes exhibited by salamanders (i.e., stepping and swimming) were evoked by electrical microstimulation ( $5-15 \mathrm{~Hz} ; 0.1-10 \mu \mathrm{A} ; 2 \mathrm{msec}$ pulses) of a circumscribed region in the caudal mesencephalon. At threshold current strength $(0.5-3.5 \mu \mathrm{A}$ at $15 \mathrm{~Hz})$, rhythmic limb movements and intersegmental coordination, such as during stepping, were induced. As the stimulation strength was subsequently increased, the frequency of stepping became more rapid, and, at 2.0-5.5 $\mu \mathrm{A}$, the limbs were held back against the body wall and swimming movements of the trunk were induced. An additional increase of the stimulation strength induced an increase of the frequency and amplitude of the swimming movements. Anatomical studies conducted in parallel revealed the presence of choline acetyltransferase immunoreactive cells in the functionally identified MLR region. Together, the present results indicate that the MLR is present in salamanders and that its level of activation determines the mode of locomotion. Walking is induced at low activation levels, and swimming, which constitutes a faster mode of locomotion, requires stronger stimulation of the MLR. Furthermore, as in other vertebrates, the MLR contains cholinergic cells.
\end{abstract}

Key words: salamander; mesencephalic locomotor region; locomotion; swimming; stepping; brainstem

\section{Introduction}

Adult salamanders are limbed lower vertebrates that spontaneously display two locomotor modes: swimming and terrestrial stepping. The two locomotor modes differ as to their patterns of activation of epaxial musculature (intersegmental coordination pattern) and limb muscles (Frolich and Biewener, 1992; Delvolvé et al., 1997). The swimming and stepping patterns of intersegmental coordination in the adult salamander are plausibly produced by the same spinal network influenced by the limb locomotor circuits, which are tonically activated during swimming and rhythmically activated during stepping (Delvolvé et al., 1997). However, to date, nothing is known on the supraspinal mechanisms, which initiate and control the stepping and the swimming patterns in the salamander and the mechanisms of switching from one locomotor mode to the other (however, see Discussion).

One region located in the midbrain from which locomotion can be initiated is the mesencephalic locomotor region (MLR) (Shik et al., 1966). It was described in several classes of vertebrates, notwithstanding the presence of different appendages or modes of locomotion. When activated, the MLR produces muscle synergies underlying stepping in mammals, flying in birds,

Received 0ct. 30, 2002; revised Dec. 13, 2002; accepted Dec. 19, 2002.

This work was supported by a group grant (Neurological Sciences) from the Canadian Institutes for Health Research (CIHR) and the Institut National de la Santé et de la Recherche Medicale INSERM-CIHR exchange program. C.B.L. received a studentship from a group grant from Le Fonds pour La Formation de Chercheurs et l'Aide à la Recherche (Québec, Canada). We thank D. Veilleux for her technical assistance.

Correspondence should be addressed to Prof. Jean-Marie Cabelguen, Institut National de la Santé et de la Recherche Médicale, Equipe 0358, Physiopathologie des Réseaux Neuronaux Médullaires, Institut François Magendie, 1 rue Camille Saint-Saëns, 33077 Bordeaux Cedex, France. E-mail: cabelguen@bordeaux.inserm.fr.

Copyright $\odot 2003$ Society for Neuroscience $\quad 0270-6474 / 03 / 232434-06 \$ 15.00 / 0$ and swimming in cyclostomes and fish (for review, see Grillner et al., 1997). Moreover, the MLR acts as a rheostat by controlling the amplitude and frequency of the locomotor movements. Descending inputs from the MLR are relayed by reticulospinal (RS) neurons located in the ventromedial medulla, and cholinergic neurons of the pedunculopontine nucleus (PPN) are part of the MLR (for review, see Jordan, 1998). The MLR of lampreys was recently identified (Sirota et al., 2000), and cholinergic inputs were also shown to be important. This study was aimed at identifying the MLR in salamanders and defining how it controls their two different modes of locomotion. Both EMG and video recordings were performed in a semi-intact salamander preparation as done in lampreys (Sirota et al., 2000). Our results show that salamanders do possess an MLR that controls two distinct patterns of locomotion (stepping and swimming), depending on its activation strength. Moreover, we report the presence of cholinergic cells within the MLR region.

\section{Materials and Methods}

Experiments were performed on 28 adult salamanders (Notophthalmus viridescens) with snout vent length (SVL) ranging from 40 to $50 \mathrm{~mm}$ $(44.9 \pm 0.1 \mathrm{~mm}$, mean $\pm \mathrm{SEM})$. The animals were obtained from the Connecticut Valley Biological Supply Company (Southampton, MA) and kept in an aquarium at room temperature. All experimental procedures conformed to the Canadian Medical Research Council guidelines and were approved by the University Animal Care Committee.

Semi-intact preparation. The animals were deeply anesthetized by immersion in a $0.1 \%$ aqueous solution of tricaine methanesulfonate (MS222; Sigma, Oakville, Ontario, Canada). Under general anesthesia, the low jaw and the forelimbs were removed, and the animal was eviscerated. The cranium and the remainder of the body, which included the 
$A_{1}$

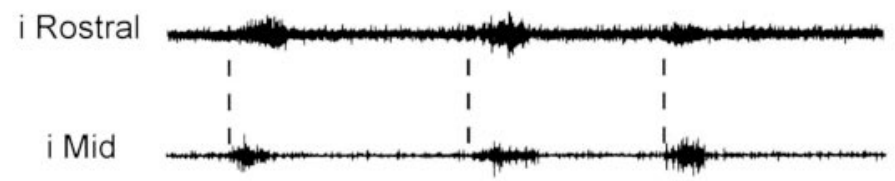

co Rostral

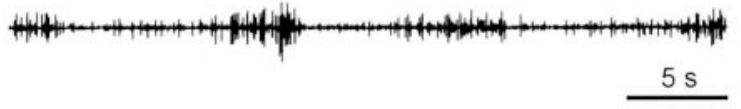

$\mathrm{B}_{1}$

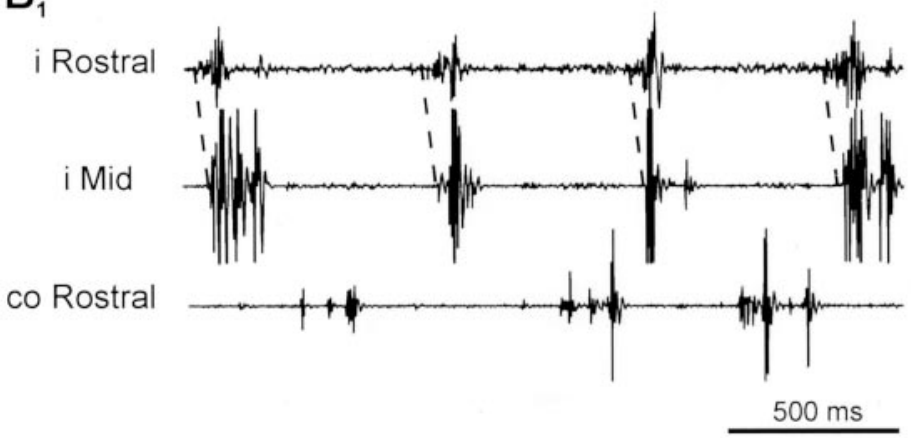

Figure 1. Stepping $\left(A_{1}, A_{2}\right)$ and swimming $\left(B_{1}, B_{2}\right)$ elicited by electrical stimulation of the same midbrain site in an adult salamander. $A_{1}, E M G$ activity recorded at $0.53 \mathrm{SVL}$ ( $i$ Rostral) and $0.76 \mathrm{SVL}$ ( $i$ Mid) on the right side and at $0.46 \mathrm{SVL}$ (co Rostral) on the left side during stepping evoked by midbrain microstimulation (2 msec pulses at $15 \mathrm{~Hz}, 2.8 \mu \mathrm{A}$ ). In-phase activation of ipsilateral myomeres is clearly visible (dashed line). $A_{2}$, Drawings illustrating the body contours every 10th successive frame (video sampling at $60 \mathrm{~Hz}$ ) during one step cycle taken from the same stepping episode. $B_{1}$, EMG activity recorded at the same segmental levels during a swimming episode evoked by microstimulation of the same midbrain site ( $2 \mathrm{msec}$ pulses at $15 \mathrm{~Hz}, 3.8 \mu \mathrm{A}$ ). A time delay between EMG bursts of ipsilateral myomeres is clearly visible (dashed line). $B_{2}$, Body movements during one swimming cycle taken from the same swimming episode.

hindlimbs and the tail, were placed in a Petri dish filled with cooled $\left(6^{\circ} \mathrm{C}\right)$ and oxygenated amphibian Ringer's solution (Delvolvé et al., 1997). While in the Petri dish, the dorsal cranium and the laminas of the first and second vertebrae were opened to expose the brain and the first two segments of the spinal cord. The optic lobes were separated into two halves with a midsagittal cut to facilitate access to the MLR. The preparation was then decerebrated by removing the forebrain rostral to the optic tectum and transferred to a recording chamber $(6.3 \times 25 \mathrm{~cm})$ superfused $(3 \mathrm{ml} / \mathrm{min})$ with cooled $\left(6^{\circ} \mathrm{C}\right)$ and oxygenated amphibian Ringer's solution. The rostral part of the preparation was stabilized by pinning down the two first vertebrae and the cranium to a thick $(1 \mathrm{~cm})$ layer of Sylgard coating the bottom half of the chamber. This configuration left the trunk caudal to the second vertebra, the hindlimbs, and the tail free to move without any contact with the bottom of the bath.

Brainstem stimulation. Glass-coated tungsten stimulating microelectrodes ( $\sim 2.5 \mathrm{M} \Omega$ impedance; $10 \mu \mathrm{m}$ tip exposed; Micro Probe, Gaithersburg, $\mathrm{MD}$ ) were used for monopolar electrical stimulation. Electrodes were angled $30^{\circ}$ anteroposteriorly, and penetrations, separated by $25 \mu \mathrm{m}$, were performed at different sites along the brainstem using a manually driven micromanipulator. At each site, the electrode was lowered into the brain tissue by $25 \mu \mathrm{m}$ steps.

A stimulator with a constant-current isolation unit was used to deliver negative pulses of $2 \mathrm{msec}$ duration at frequencies of $5-15 \mathrm{~Hz}$. At each stimulated site, the current was increased by steps of 0.1 up to $10 \mu \mathrm{A}$. Typically, a few search trials were performed with continuous stimulation not exceeding $30 \mathrm{sec}$. A rest period of $\geq 1 \mathrm{~min}$ was allowed between successive trials. Once the MLR was localized, we did not stimulate for $>30 \mathrm{sec}$ in any one trial because of concerns about damaging the stimulation site. For later histological identification, an electrolytic lesion $(n=12)$ was made at the stimulation site using DC current $(5 \mu \mathrm{A})$ applied for $10 \mathrm{sec}$ at the end of experiments.

EMG recording. EMG recordings were made in 11 animals with pairs of

$\mathrm{A}_{2}$

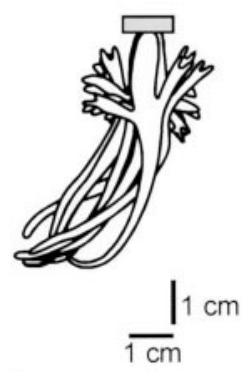

$\mathrm{B}_{2}$

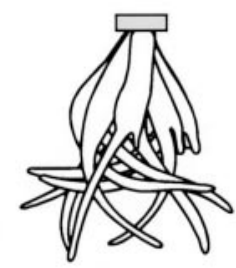

insulated stainless steel wires (diameter of 50 $\mu \mathrm{m}$; California Fine Wire, Grover Beach, CA) with bared ends (exposure $<0.5 \mathrm{~mm}$ ) inserted through the skin into myomeres of the musculus dorsalis trunci. The electrode tips were separated by $<1 \mathrm{~mm}$, and their rostrocaudal position was expressed as a fraction of the SVL. The voltage signals from electrodes were differentially amplified 10,000 times (bandpass of 30$300 \mathrm{~Hz}$ with a $60-\mathrm{Hz}$ notch filter), displayed on an oscilloscope, and stored on magnetic tape.

Video recording. In two experiments, the motor responses of the animal were videotaped with a camera positioned $1 \mathrm{~m}$ above the bath. Each video frame was captured (Northern Exposure software; Empix Imaging, Mississauga, Ontario, Canada) to draw the body shape of the salamander. This method allowed us to characterize the induced motor behaviors.

Anatomical studies. The distribution of cholinergic neurons in the isthmic region of salamanders $(n=17 ; 12$ animals with an electrolytic lesion in the MLR and five unlesioned) was studied using immunohistochemistry for choline acetyltransferase (ChAT). The fixed brains in $4 \%$ paraformaldehyde in $0.1 \mathrm{~m}$ phosphate buffer, $\mathrm{pH} 7.4$, were frozen and cut at 20 $\mu \mathrm{m}$ thickness on a cryostat (American Optical, Buffalo, NY) or embedded in agarose and cut at $40 \mu \mathrm{m}$ thickness on a vibratome (TPI, St. Louis, MO). The sections (mounted on gelatinized slides or free-floating sections, respectively) were then processed for immunohistochemistry using a procedure adapted from Marin et al. (1997). A preincubation period of $2 \mathrm{hr}$ was added before the primary antibody step, and we replaced the peroxidase-antiperoxidase method by the avidin-biotin method. As a control, some sections were processed without the primary antiserum, which resulted in no specific labeling of somata and fibers.

\section{Results}

After a recovery period of $1-2 \mathrm{hr}$, all preparations were reactive to touching of the head or gentle squeezing of the tail or the hindlimbs. Most of them $(n=15)$ displayed some spontaneous rhythmic movements of the trunk and/or hindlimbs, but these movements were infrequent, and sequences were rarely longer than a few cycles. Therefore, they were not analyzed. Quantitative analyses were performed only on animals in which EMG recordings were made $(n=11)$.

For a typical experiment, repetitive $(15 \mathrm{~Hz})$ electrical stimulation of a mesencephalic region lying just rostral to the isthmus induced two distinct rhythmic motor patterns depending on the current intensity (Fig. 1). At low current intensity $(2.8 \mu \mathrm{A})$, the motor pattern was characterized by an in-phase activation of the ipsilateral trunk myomeres and an alternated activation of left and right trunk myomeres (Fig. $1 A_{1}$ ). The video recordings also showed that the electrical stimulation evoked small-amplitude rhythmic lateral displacements of the midtrunk and rhythmic alternated movements of the hindlimbs (Fig. $1 A_{2}$ ). As the current intensity was subsequently increased to $3.8 \mu \mathrm{A}$, the induced EMG pattern changed dramatically, as did the movement pattern. The frequency and the amplitude of the EMG bursts increased markedly, and there was a rostrocaudal activation of trunk myomeres on the same side of the body, although left and right trunk myomeres were still activated in alternation (Fig. 

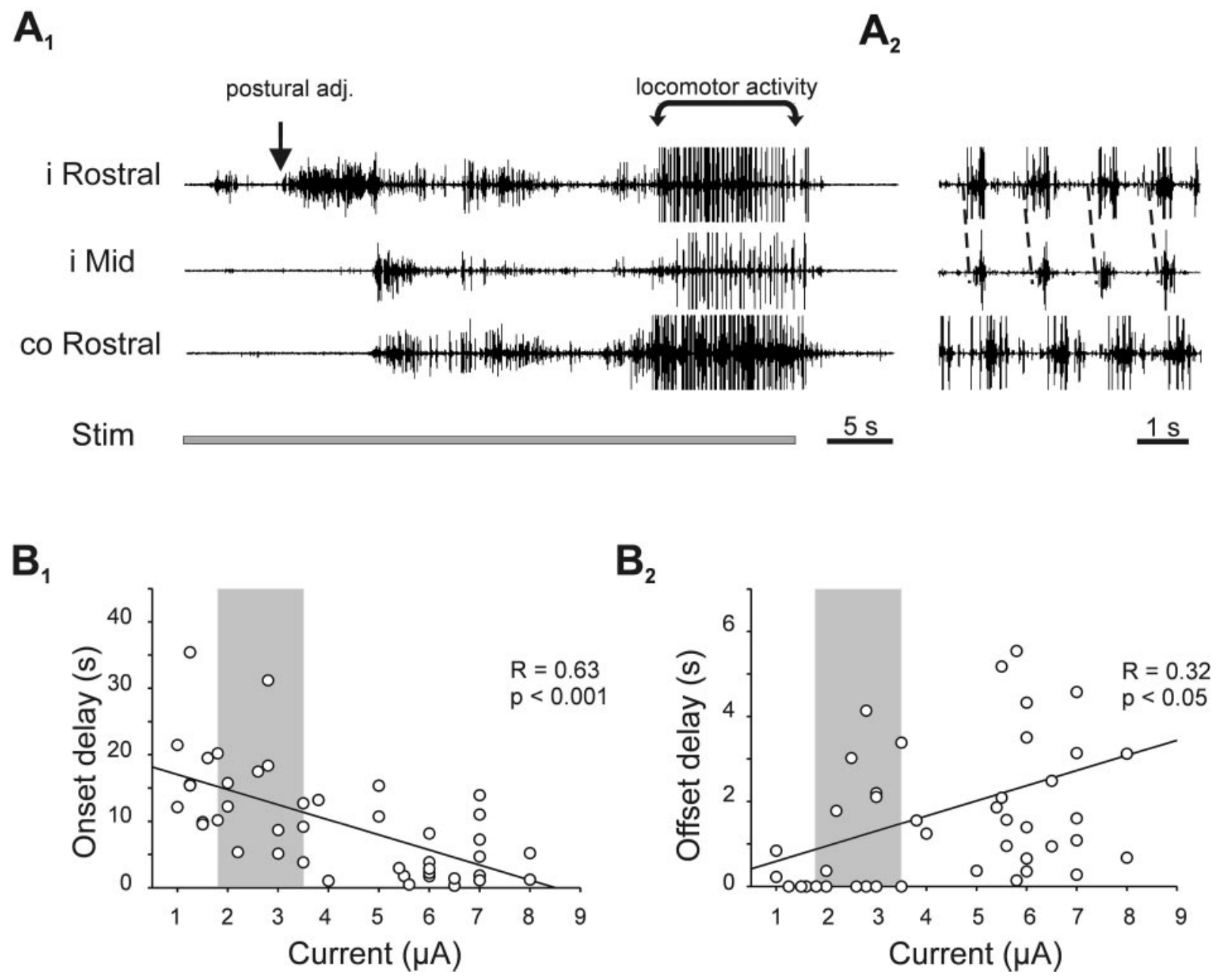

Figure 2. Initiation and termination of MLR-induced locomotion. $A_{1}$, A period of postural adjustment began ( arrow) a few seconds after the onset of MLR stimulation and lasted $\sim 25$ sec before the locomotion started (locomotor activity). The gray bar at bottom indicates the period during which the MLR was stimulated ( 2 msec pulses at $15 \mathrm{~Hz}, 2.5 \mu \mathrm{A}$ ). $A_{2}$, Expansion of a part of the locomotor episode shown in $A_{1}$. The rostrocaudal phase lag between EMG bursts of ipsilateral myomeres (dashed line) and the alternation between ipsilateral and contralateral EMG bursts are clearly visible. Same voltage amplification in $A_{1}$ and $A_{2}$. $B_{1}$, Relationship between the onset delay and the stimulus intensity. $B_{2}$, Relationship between the end ("offset") delay and the stimulation intensity. In $B_{1}$ and $B_{2}$, data are from the same four animals. The gray areas indicate the range of stimulus intensities that evoked stepping and/or swimming.

$\left.1 B_{1}\right)$. The body and tail displayed rhythmic lateral displacements of large amplitude, whereas the hindlimbs were held back against the body wall (Fig. $1 B_{2}$ ).

Similar EMG and movement patterns have been reported previously during overground stepping and swimming, respectively, in the intact salamander (Frolich and Biewener, 1992; Delvolvé et al., 1997). In the present study, similar results were obtained in 7 of 11 animals with the threshold current for inducing stepping ranging from 0.5 to $3.5 \mu \mathrm{A}(2.26 \pm 0.5 \mu \mathrm{A})$ and that for inducing swimming between 1.8 and $5.5 \mu \mathrm{A}(3.47 \pm 0.51 \mu \mathrm{A})$. Furthermore, the range of intensities that evoked stepping was smaller (usually $1 \mu \mathrm{A}$ at $15 \mathrm{~Hz}$ ) than that which evoked swimming $(3-5 \mu \mathrm{A}$ at $15 \mathrm{~Hz})$.

The effective sites of stimulation were found on either side in the isthmic region, close to the midline, extending $\sim 100 \mu \mathrm{m}$ rostrocaudally. A good indication that the electrode was located in the effective zone was the observed stiffness of the trunk produced before locomotor movements. This postural change was related directly to the EMG pattern displayed by myomeres before the MLR-induced locomotor episode. Typically, a burst of EMG activity occurred in the rostral myomeres ipsilateral to the stimulation side, followed by a bilateral sustained EMG activity along the body (Fig. $2 A_{1}$ ). Thereafter, the EMG pattern changed dramatically to a locomotor pattern characterized by vigorous EMG discharges during swimming (Fig. $2 \mathrm{~A}_{2}$ ).

In four animals, the effective sites were not successfully identified, probably because of the very small size of the region and/or the damage that may have resulted from the multiple electrode penetrations in the region containing the effective sites. The extent of spread of the current injection was not tested, but moving the electrode away $(25 \mu \mathrm{m})$ from an effective site led to disappearance of locomotion. Moreover, stimulation of slightly more rostral or lateral sites produced different motor responses (e.g., axial twisting and rhythmic movements of the tip of the tail or of only one hindlimb) or was not effective.

MLR-evoked locomotion (stepping or swimming) usually continued throughout the stimulation duration (up to $30 \mathrm{sec}$ ). However, signs of adaptation were sometimes observed (i.e., both the frequency and the amplitude of the evoked EMG bursts decreased and eventually locomotion stopped). The delay between the onset of stimulation and the onset of the MLR-evoked locomotion depended on the animal, but, for all individuals, it was always shorter for higher stimulation intensities. In four animals, we quantified the relationship between the onset delay and the current strength at a fixed frequency of stimulation $(15 \mathrm{~Hz})$. Fig- 

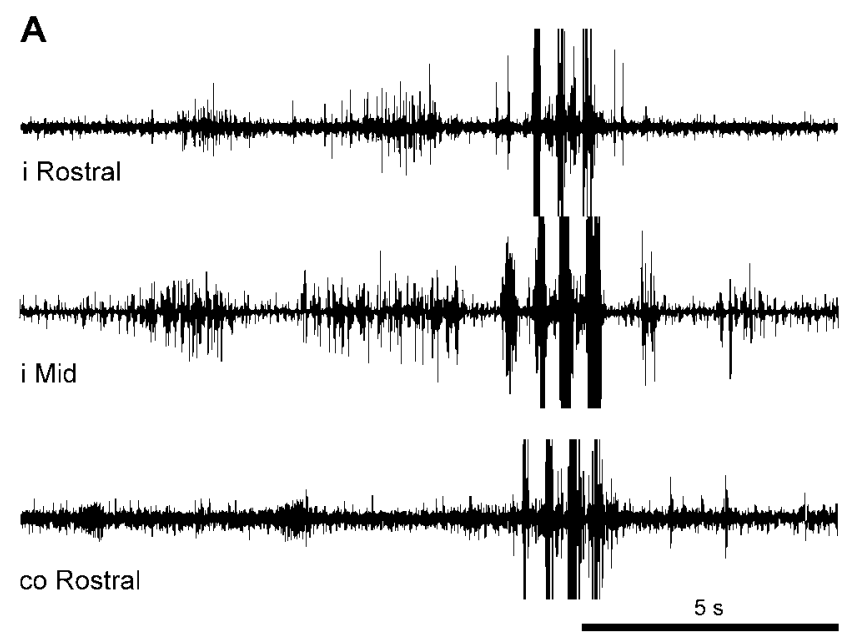

$B_{1}$
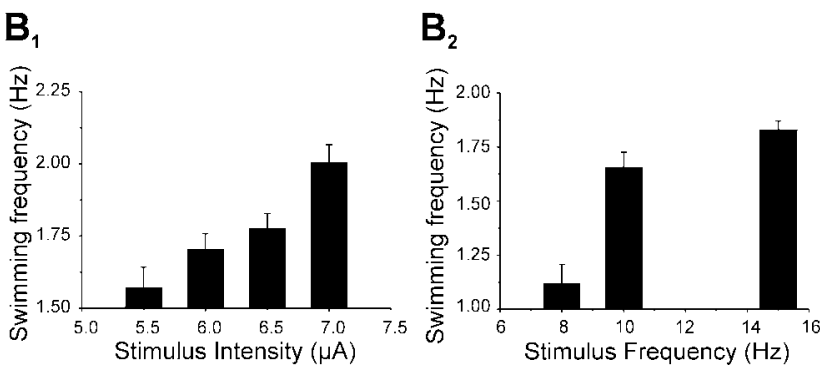

Figure 3. MLR stimulation induces two modes of locomotion in salamander. $A$, Traces are, from top to bottom, EMG activity recorded at 0.39SVL ( $i$ Rostral) and 0.74 SVL (i Mid) on the right side and $0.41 \mathrm{SVL}$ (co Rostral) on the left side. Electrical stimulation of the MLR ( 2 msec pulses at $15 \mathrm{~Hz}, 1.25 \mu \mathrm{A}$ ) started $2.2 \mathrm{sec}$ before recordings and was maintained $30 \mathrm{sec}$. Note the abrupt switching from stepping to swimming and back to stepping. $B_{1}$, Effect of increasing the stimulus intensity (2 msec pulses at $15 \mathrm{~Hz}$ ) on the frequency of swimming. $B_{2}$, Effect of increasing the stimulus frequency ( $2 \mathrm{msec}$ at $6 \mu \mathrm{A})$ on the frequency of swimming. Bars are mean values, and error bars are SEM.

ure $2 B_{1}$ shows that there was a significant negative correlation $(r=0.63 ; p<0.001)$ between the onset delay and the stimulus strength. Locomotion usually ceased on termination of the stimulation except at the highest current intensities (i.e., during fast swimming), at which it outlasted the stimulation period by a few seconds. A weak but significant positive correlation $(r=0.32 ; p<$ 0.05 ) was found between the offset delay and the current strength in data pooled from four individuals (Fig. $2 \mathrm{~B}_{2}$ ).

In some cases, during constant electrical stimulation at intensity above the stepping threshold, a stepping pattern occurred first and then switched spontaneously in only one cycle to a swimming one (Fig. 3A). Swimming lasted a few cycles and switched back to a stepping pattern in one cycle.

During evoked stepping, the frequency and the amplitude of the EMG bursts depended on the stimulation strength intensity. Increasing the current level from 2.8 to $3.5 \mu \mathrm{A}$ (at $15 \mathrm{~Hz}$ ) increased the intensity of the individual EMG bursts and also doubled the stepping rhythm $(0.08-0.16 \mathrm{~Hz})$. The amplitude and the frequency of the EMG bursts during swimming were also related to the intensity of stimulation. The frequency of bursting (Fig. $3 B_{1}$ ) increased from $1.57 \pm 0.07$ to $2.01 \pm 0.06 \mathrm{~Hz}$ when the current level was increased from 5.5 to $7 \mu \mathrm{A}$ (at $15 \mathrm{~Hz}$ ). Likewise, at a constant stimulating current ( $6 \mu \mathrm{A}$ in this experiment), the swimming rhythm increased $\left(1.12 \pm 0.09\right.$ to $1.83 \pm 0.04 \mathrm{~Hz}$ ) (Fig. $\left.3 B_{2}\right)$ as the stimulation frequency increased $(8-15 \mathrm{~Hz})$. In this example, swimming switched to stepping when the frequency of $6 \mu \mathrm{A}$ amplitude pulses was lowered to $7 \mathrm{~Hz}$, and, as the stimulating frequency was lowered more, the stepping pattern waned to a stop for stimulation frequency $<5 \mathrm{~Hz}$ (data not illustrated).

Therefore, it could be concluded that the amplitude and frequency of the EMG bursts during evoked locomotion (stepping or swimming) could be controlled by the strength of the stimulation.

There is evidence that cholinergic cells are located in the physiologically identified MLR of several vertebrate species (Grillner et al., 1997). To determine whether cholinergic cells are present within the functionally identified MLR, immunohistochemistry against ChAT was used after an electrolytic lesion was made at the stimulation site. The coagulated site (Fig. 4A) was found to be surrounded by numerous strongly ChAT-IR fibers and few ovoid ChAT-IR cells (Fig. $4 B$, arrows). The immunohistochemical reaction was also performed on intact brainstems $(n=5)$ and allowed us to identify the cholinergic cell groups in the MLR region (Figs. $4 C, D, 5$ ). There were two distinct groups of ChAT-IR cell bodies in the isthmic tegmentum, as described previously by Marin et al. (1997). A first group consisted of numerous strongly labeled cells (Figs. 4D, 5). Their axons formed a conspicuous bundle that could be followed as far as the rostral mesencephalon (Fig. 4D). This group of ChAT-IR cells corresponds to the laterodorsal tegmental nucleus (LDT) of other vertebrates, including mammals and lampreys. A second, smaller group was located more rostrally and more laterally in the tegmentum and corresponds to the nucleus isthmi (NI) (Figs. 4D, 5 ). The same cellular organization seen in Figure $4, B$ and $D$, indicates that the salamander MLR is located in the rostral part of the LDT. A third isthmic small, compact group of ChAT-IR cells dorsal and rostral to the NI was described in Pleurodeles walt (Marin et al., 1997), but, according to our observations, it is absent in $N$. viridescens.

\section{Discussion}

Two main conclusions can be drawn from the present study: (1) the MLR is present in salamanders, and (2) depending on its strength, the MLR activation induces two distinct locomotor patterns (stepping and swimming). It is noteworthy that walking occurs at low intensity, whereas swimming requires higher intensities. This is consistent with swimming being the fastest mode of locomotion in these animals, which are mechanically better adapted for swimming than walking because of their sprawling posture and their short limbs (Ashley-Ross, 1994).

For both walking and swimming, the locomotor rhythm was always slower than the locomotor rhythms in intact salamanders (Frolich and Biewener, 1992; Delvolvé et al., 1997). This probably resulted from different external conditions between intact locomoting animals and the present decerebrated animals, which were partly restrained with their hindlimbs not touching the ground. Indeed, previous study in cats has shown that the frequency of the locomotor movements induced by MLR stimulation depends both on the frequency and intensity of stimulation of the MLR and on external conditions (Shik et al., 1966).

Previous behavioral observations in decerebrated Necturus have shown that electrical stimulation of the lateral mesencephalic tegmentum can induce rhythmic movements of the body, the tail, and the limbs in different combinations (Shik, 1997). At variance with our results, the rhythmic movements involving body, tail, and limbs rather than those involving body and tail were evoked at higher stimulation strengths. Although it is likely that some of the evoked movements corresponded to the locomotor EMG patterns evidenced in the present study, behavioral 

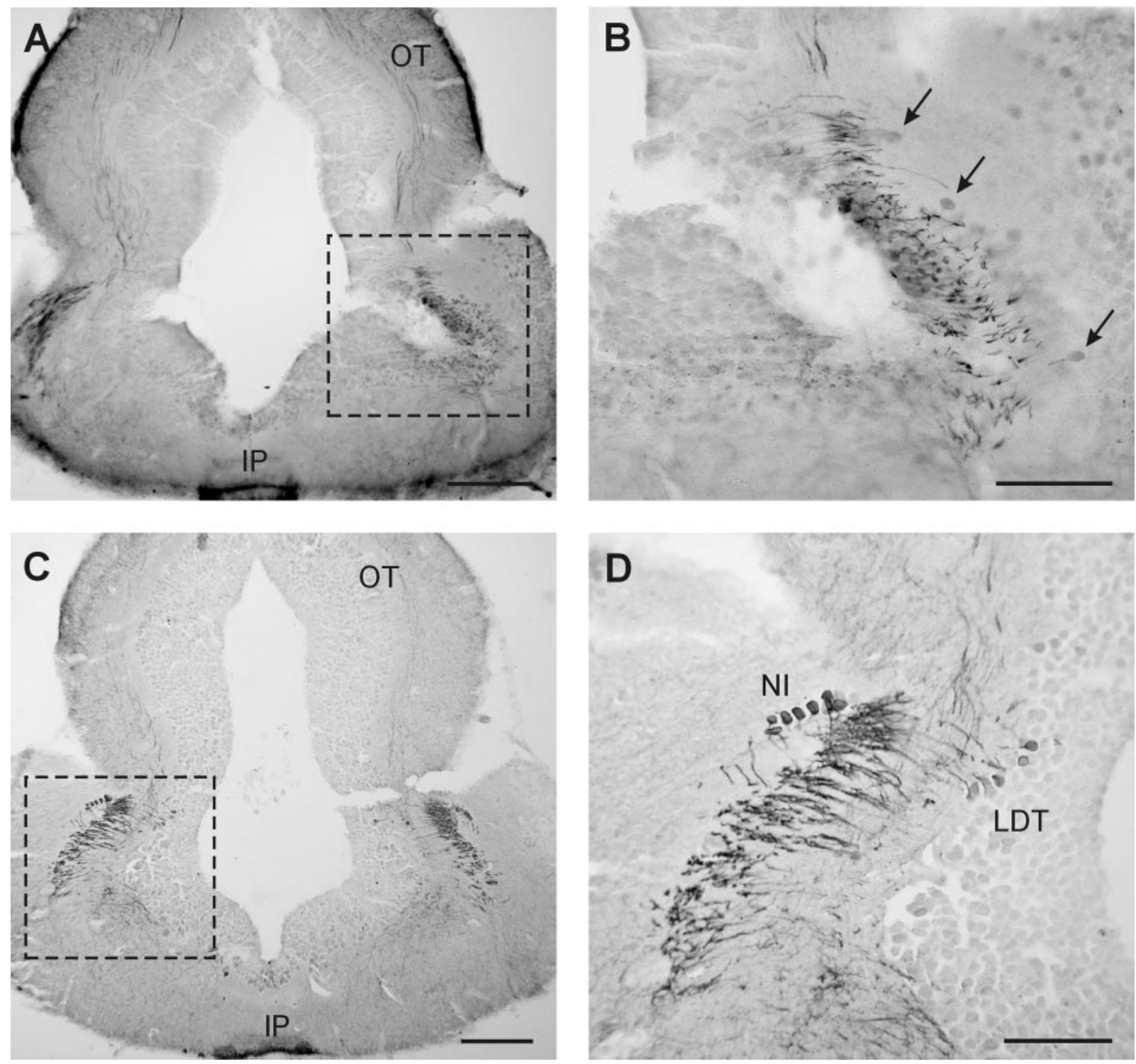

Figure 4. ChAT-IR cells around the MLR-coagulated site. $A$, Transverse section ( $40 \mu \mathrm{m}$ thick) at the isthmic level showing the coagulated site (right) and the intact side (left). Note the dense plexus of ChAT-IR fibers in the interpeduncular nucleus. B, Magnification of the boxed area in A showing ovoid ChAT-IR cells (arrows) and numerous ChAT-IR fibers around the coagulated site. C, Transverse section (20 $\mu \mathrm{m}$ thick) at approximately the same level as in $A$. The interpeduncular nucleus shows intense labeling. D, Magnification of the boxed area in ( showing two distinct groups of ChAT-IR cells, the LDT and the NI. Note the axonal tract arising from the caudal LDT. Scale bars: $A, C, 200 \mu \mathrm{m} ; B, D, 100 \mu \mathrm{m}$. IP, Interpeduncular nucleus; $0 T$, optic tectum.

observations alone did not permit discrimination between uncoordinated rhythmic movements and genuine locomotion.

The present study also shows that the effective sites of stimulation were located within a specific and relatively small region at the mesopontine border. The MLR is a structure that was defined functionally rather than anatomically. Physiological and pharmacological observations made in mammals indicate that cell bodies and not passing axons constitute the MLR. Our data did not allow us to determine whether the neural elements activated were passing axons and/or neurons. However, stimulation of the rostral midbrain did not evoke locomotion, suggesting that there is not a more rostral nucleus projecting through the region that we now define as the MLR of salamanders and that is responsible for the locomotor movements observed. Therefore, a subset of this area must contain the cell bodies of origin for descending pathways responsible for initiating locomotion. In higher vertebrates, local electrical stimulation in different parts of the MLR generates various locomotor behaviors, suggesting that this region is heterogeneous (Sinnamon, 1993). Indeed, it has been established that the cuneiform nucleus and the PPN are the main components of the mammalian MLR (Grillner et al., 1997) (for review, see Jordan, 1998). In the rat, a small proportion of cholinergic neurons of the PPN and the LDT project specifically to the reticular formation of the ventromedial medulla (Skinner et al., 1990). Recently, it was demonstrated that MLR inputs to lamprey RS cells are at least partly cholinergic (Brocard et al., 2001). Moreover, two distinct groups of cholinergic neurons were described in the lamprey mesopontine tegmentum, the LDT and the PPN (Pombal et al., 2001). As in lampreys, the present results indicate that cholinergic neurons of the LDT are a structural component of the MLR of salamanders. Within the class of lower vertebrates, significant differences exist in the organization of the cholinergic groups at the isthmic level. Indeed, in our study, only the LDT is present in salamander $N$. viridescens, whereas the PPN is absent. 


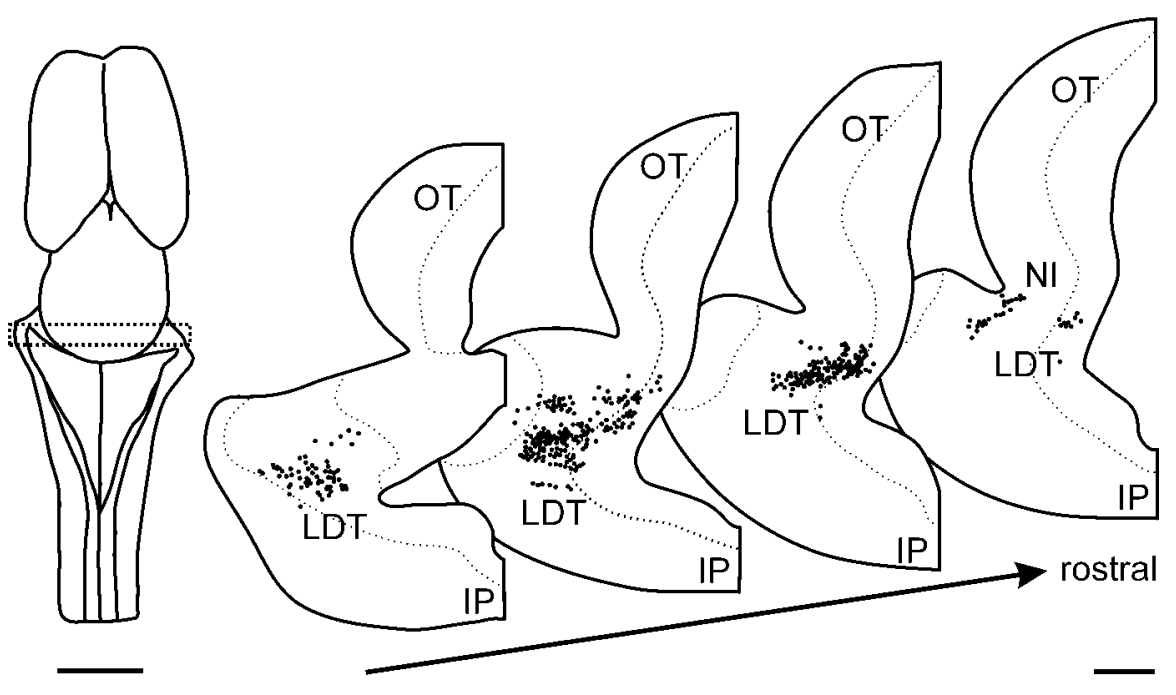

Figure 5. Schematic representation of the distribution of ChAT-IR cells at the isthmic level. Each schematic represents three consecutive $20-\mu \mathrm{m}$-thick sections. Total distance, $240 \mu \mathrm{m}$. Scale bars: whole brain representation, $1 \mathrm{~mm}$; brainstem transverse sections, $200 \mu \mathrm{m}$. IP, Interpeduncular nucleus; $0 T$, optic tectum.

Nevertheless, Marin et al. (1997) described some clustered ChAT-IR cells rostral and dorsal to the nucleus isthmi in Pleurodeles waltl species, which they proposed as perhaps corresponding to the PPN.

There is anatomical and electrophysiological evidence in several species that the MLR activates the spinal pattern generators for locomotion through an activation of RS neurons (for review, see Orlovsky et al., 1999). Therefore, it is likely that the descending influences from the MLR on the spinal locomotor networks in the salamander also involve brainstem RS neurons. More anatomical and electrophysiological studies are needed to establish the detailed connectivity between MLR and specific groups of RS neurons in salamander. Interestingly, we have preliminary data with iontophoretically injecting the retrograde tracer cobaltlysine in the pontine reticular formation, indicating that some of the cholinergic neurons from the MLR could project to the rhombencephalic reticular formation. Furthermore, hindbrain neurons, which might participate in the initiation of locomotion induced by mesencephalic stimulation, have been studied electrophysiologically in rough-skin newts (Bar-Gad et al., 1999). Additional experiments are nevertheless needed to determine their connectivity, intrinsic membrane properties, relationships with the spinal locomotor networks, and involvement in switching between two locomotor modes. Long-lasting plateau potentials in RS neurons are responsible for the onset of swimming in response to brief skin stimulations in lampreys (Viana Di Prisco et al., 1997, 2000). Whether similar plateau properties are involved in both MLR-induced locomotor modes or only one of them (e.g., swimming) in salamander remains to be determined.

It is unknown whether or not the activated target RS cells involved are different during MLR-induced stepping and swimming. One possibility is that stepping and swimming involve specific populations of RS cells and that the switch to swimming results simply from an additional recruitment of groups of RS neurons. However, because at a specific stimulation strength, locomotion could sometimes spontaneously switch from stepping to swimming and back to stepping, this hypothesis seems less likely. Another possibility is that the transition between the two locomotor modes may result from a reconfiguration of the same spinal network by RS modulatory influences. Hence, the abrupt switch from stepping to swimming would be explained by modifications of cellular properties and/or intercellular connections within that spinal network. Interestingly, it has been proposed previously that the axial motor patterns during swimming and stepping are both generated by the same, essentially lamprey-like, network that receives different input drives from the limb locomotor networks, depending on the locomotor mode (Delvolvé et al., 1997).

In conclusion, we showed that salamanders, like other vertebrates, do have a specific mesencephalic locomotor region that controls the initiation of locomotion and that the level of activation of that region determines the mode of locomotion. The presence of such a region with characteristics similar to that found in other vertebrates provides additional evidence that the mechanisms of brainstem control of locomotion are well preserved throughout the vertebrate phylum. Moreover, a cholinergic component from the MLR is most likely involved in the initiation of locomotion, as in mammals and lampreys.

\section{References}

Ashley-Ross M (1994) Hindlimb kinematics during terrestrial locomotion in a salamander (Dicamptodon tenebrosus). J Exp Biol 193:255-283.

Bar-Gad I, Kagan I, Shik ML (1999) Behavior of hindbrain neurons during the transition from rest to evoked locomotion in a newt. Prog Brain Res 123:285-294.

Brocard F, Bourcier-Lucas C, LeRay D, Dubuc R (2001) Inputs from the mesencephalic locomotor region to reticulospinal neurons in lampreys. Soc Neurosci Abstr 27:306.4.

Delvolvé I, Bem T, Cabelguen JM (1997) Epaxial and limb muscle activity during swimming and terrestrial stepping in the adult newt, Pleurodeles waltl. J Neurophysiol 78:638-650.

Frolich LM, Biewener AA (1992) Kinematic and electromyographic analysis of the functional role of the body axis during terrestrial and aquatic locomotion in the salamander Ambystoma tigrinum. J Exp Biol 162:107-130.

Grillner S, Georgopoulos AP, Jordan LM (1997) Selection and initiation of motor behaviour. In: Neurons, networks and motor behavior (Stein PSG, Grillner S, Selverston A, Stuart DG, eds), pp 4-19. Cambridge, MA: MIT.

Jordan LM (1998) Initiation of locomotion in mammals. Ann NY Acad Sci 860:83-93.

Marin O, Smeets WJ, Gonzalez A (1997) Distribution of choline acetyltransferase immunoreactivity in the brain of anuran (Rana perezi, Xenopus laevis) and urodele (Pleurodeles waltl) amphibians. J Comp Neurol 382:499-534.

Orlovsky GN, Deliagina TG, Grillner S (1999) Neural control of locomotion: from mollusc to man, pp 205-214. New York: Oxford UP.

Pombal MA, Marin O, Gonzalez A (2001) Distribution of choline acetyltransferase-immunoreactive structures in the lamprey brain. J Comp Neurol 431:105-126.

Shik ML (1997) Locomotor patterns elicited by electrical stimulation of the brain stem in the mudpuppy. Motor Control 1:354-358.

Shik ML, Severin FV, Orlovsky GN (1966) Control of walking and running by means of electrical stimulation of the midbrain. Biophysics 11:756-765.

Sinnamon HM (1993) Preoptic and hypothalamic neurons and the initiation of locomotion in the anesthetized rat. Prog Neurobiol 41:323-344.

Sirota MG, Viana Di Prisco G, Dubuc R (2000) Stimulation of the mesencephalic locomotor region elicits controlled swimming in semi-intact lampreys. Eur J Neurosci 12:4081-4092.

Skinner RD, Kinjo N, Ishikawa Y, Biedermann JA, Garcia-Rill E (1990) Locomotor projections from the pedunculopontine nucleus to the medioventral medulla. NeuroReport 1:207-210.

Viana Di Prisco G, Pearlstein E, Robitaille R, Dubuc R (1997) Role of sensoryevoked NMDA plateau potentials in the initiation of locomotion. Science 278:1122-1125.

Viana Di Prisco G, Pearlstein E, Le Ray D, Robitaille R, Dubuc R (2000) A cellular mechanism for the transformation of a sensory input into a motor command. J Neurosci 20:8169-8176. 